\title{
Neurogenesis in an Adult Avian Song Nucleus Is Reduced by Decreasing Caspase-Mediated Apoptosis
}

\author{
Christopher K. Thompson ${ }^{1}$ and Eliot A. Brenowitz ${ }^{1,2,3}$ \\ ${ }^{1}$ Graduate Program in Neurobiology and Behavior, and Departments of ${ }^{2}$ Psychology and ${ }^{3}$ Biology, University of Washington, Seattle, Washington $98195-1525$
}

\begin{abstract}
Neuron death and replacement are fundamental components of brain plasticity. Much remains unknown, however, about the mechanistic interaction between neuron death and neurogenesis in adult vertebrates. In seasonally breeding adult male white-crowned sparrows, the song system nucleus HVC loses $\sim 26 \%$ of its neurons via caspase-dependent apoptosis within $4 \mathrm{~d}$ after a transition to nonbreeding physiological conditions. To determine whether neuronal death is necessary for the recruitment of new neurons, we infused caspase inhibitors into HVC in vivo and suppressed neurodegeneration for at least $20 \mathrm{~d}$ after the transition to nonbreeding conditions. The blockade of HVC neuron death reduced the number and density of new neurons recruited to the ipsilateral HVC by 48 and $29 \%$, respectively, compared with contralateral HVC. Our results are the first to show that reducing neuronal death in the adult brain decreases the recruitment of new neurons.
\end{abstract}

\section{Introduction}

The birth and death of neurons are fundamental processes in the development and adult plasticity of the vertebrate brain. Although ongoing neurogenesis in the postnatal brains of endothermic vertebrates was first suggested by Altman and colleagues over 40 years ago (Altman and Das, 1965) and conclusively demonstrated by Nottebohm and colleagues (Goldman and Nottebohm, 1983; Paton and Nottebohm, 1984), many fundamental questions about the mechanisms of neuronal replacement remain. Of particular importance is the relationship between the rate of neuron death and the rate of neurogenesis. Given that the brains of endothermic vertebrates do not continue to grow without limit, the addition of new neurons to the adult brain must be offset by the death of mature neurons. There is an extraordinary exception to this generalization, however. In seasonally breeding male songbirds, the avian song control system (see Fig. 1A), a series of discrete brain nuclei that regulates the production and memorization of song in songbirds, undergoes dramatic morphological and physiological change across seasons, which are largely driven by changes in circulating levels of testosterone $(\mathrm{T})$ and its metabolites in the brain. Neuron number in the song system nucleus HVC (used as a proper name) can vary by as much as $50 \%$ across seasons in some species (Brenowitz et al., 1991). The rate of neuronal recruitment into HVC (acronym used as the proper name) is highest under nonbreeding conditions, when HVC is regressed and total HVC neuron number (i.e., new + mature neurons) is lowest (Kirn et al., 1994; Tramontin and Brenowitz, 1999). In addition, targeted photolysis of a

Received Nov. 10, 2008; revised March 3, 2009; accepted March 5, 2009

This work was supported by National Institutes of Health Grants MH53032, P30DC04661, DC03829, and 5 T32GM07108. We thankE. Rubel, K. Lent, and G. MacDonald for technical assistance and advice.

Correspondence should be addressed to Christopher K. Thompson at his present address: Freie Universität Berlin, Institut für Biologie, 14195 Berlin, Germany. E-mail: ckt@zedat.fu-berlin.de.

DOI:10.1523/JNEUROSCI.5423-08.2009

Copyright $\odot 2009$ Society for Neuroscience $\quad$ 0270-6474/09/294586-06\$15.00/0 specific population of HVC neurons increases the rate of neuronal recruitment (Scharff et al., 2000). These results demonstrate that in brain circuits that undergo neuronal turnover, the death of existing neurons is sufficient to increase the rate of incorporation of new neurons. Whether neuron death is necessary for neuronal recruitment has not been directly addressed, however.

Naturally occurring seasonal regression of HVC morphology, largely driven by a loss of HVC neurons, serves as an excellent model in which to examine the relationship between neuron death and recruitment. Both HVC volume and neuron number increase in male white-crowned sparrows (Zonotrichia leucophrys Gambelii) exposed to breeding conditions in a laboratory setting (Smith et al., 1995). A rapid transition to nonbreeding conditions induces rapid regression of the song control system, including significant regression of $\mathrm{HVC}$ volume within $12 \mathrm{~h}$ and loss of $\sim 26 \%$ of neurons within $4 \mathrm{~d}$ (Thompson et al., 2007). HVC regression is dependent upon apoptotic-like mechanisms; infusion of a mixture of caspase inhibitors via a cannula and osmotic pump suppresses caspase-dependent neurodegeneration in HVC and prevents the reduction in $\mathrm{HVC}$ volume and neuron number normally observed after a transition to nonbreeding conditions (Thompson and Brenowitz, 2008).

To determine whether the increase in neuron death that occurs during the transition to nonbreeding conditions is necessary for the subsequent increase in neuronal recruitment, we infused caspase inhibitors near HVC on one side of the brain of wildcaught male white-crowned sparrows. We found that continuous caspase inhibitor infusion protected the ipsilateral HVC from neurodegeneration and reduced the density and number of new neurons incorporated into HVC, a result that supports the hypothesis that neuron death is necessary for subsequent neuronal recruitment.

\section{Materials and Methods}

All procedures followed National Institutes of Health animal use guidelines and were approved by the University of Washington Institutional 


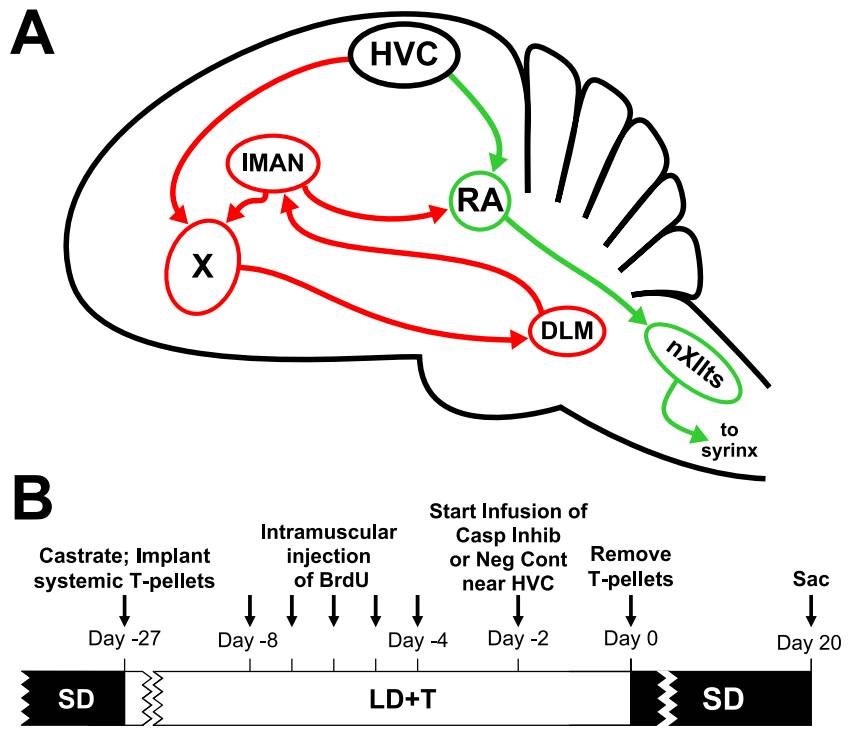

Figure 1. The avian song control system and timeline for experimental procedures. $A$, Schematic sagittal diagram of the song control system showing projections of major nuclei. The green arrows indicate the descending motor pathway. The red arrows indicate the anterior forebrain pathway. X, Area X; IMAN, lateral magnocellular nucleus of the anterior nidopallium;

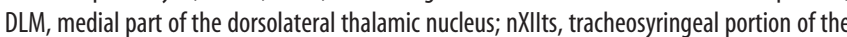
hypoglossal nucleus. $B$, Schematic timeline of the experimental procedures. Birds received daily injections of BrdU for $5 \mathrm{~d}$.

Animal Care and Use Committee. We captured 10 male Gambel's whitecrowned sparrows in eastern Washington during their postbreeding season migration. We housed the birds in indoor group aviaries for at least 10 weeks on short-day photoperiod (SD; $8 \mathrm{~h}$ light) before the start of the experiment to ensure that the song system was sufficiently regressed and sensitive to the stimulatory effects of laboratory breeding conditions [exposure to long-day photoperiod (LD) and elevated levels of circulating $\mathrm{T}$ $(\mathrm{LD}+\mathrm{T})]$.

At the beginning of the experiment we transferred the birds from light $(\mathrm{L}) /$ dark $(\mathrm{D})=8 / 16(\mathrm{SD})$ to $\mathrm{L} / \mathrm{D}=20 / 4(\mathrm{LD})$ overnight (see Fig. $1 B$ for timeline of experimental procedures). The same day we castrated each bird while anesthetized with isoflurane through a non-rebreathing system by aspiration of the testes. Once castrated, we implanted each bird subcutaneously with a $12 \mathrm{~mm}$ SILASTIC capsule filled with crystalline T. We castrated the birds at the onset of LD to eliminate the burden of multiple surgeries when birds are implanted with cannulae at the transition from nonbreeding to breeding conditions. We housed the birds individually in visual and auditory contact with the other birds used in this experiment. We kept all the birds under $\mathrm{LD}+\mathrm{T}$ for $28 \mathrm{~d}$, which is sufficient to induce full growth of the song control system under these conditions (Tramontin et al., 2000).

After $20 \mathrm{~d}$ of $\mathrm{LD}+\mathrm{T}$, we injected 5-bromodeoxyuridine (BrdU; 15 $\mathrm{mg} / \mathrm{ml}$ in $0.09 \% \mathrm{NaCl}$ and $0.012 \mathrm{M} \mathrm{NaOH}$; Sigma) intramuscularly at 50 $\mathrm{mg} / \mathrm{kg}$ once a day for $5 \mathrm{~d}$. BrdU is a thymidine analog that is incorporated into newly generated DNA of cells undergoing mitosis and is used here as a marker to identify new cells.

After $26 \mathrm{~d}$ of LD + T conditions, we implanted a cannula attached to an osmotic pump unilaterally near HVC in each bird. Given that there are no direct connections between HVC on the two sides of the brain, unilateral infusion of caspase inhibitors leaves the untreated contralateral hemisphere to serve as a within-animal control. We anesthetized each bird with isoflurane and placed its head into a stereotaxic holder. We made an incision into the scalp, removed the portion of the skull overlying the midsagittal sinus, and used the bifurcation of the midsagittal sinus as a zero point for the cannula. We randomly chose a hemisphere and lowered the cannula $0.75 \mathrm{~mm}$ into the telencephalon caudal to HVC (lateral, $2.8 \mathrm{~mm}$; posterior, $0.4 \mathrm{~mm}$ ). We fixed the cannula to the skull with dental cement and attached an osmotic pump (Alzet model 1002) to the cannula. Six birds received osmotic pumps filled with a mixture of caspase inhibitors $(0.015 \mathrm{mg}$ in $100 \mu \mathrm{l}$ in $1 \%$ DMSO in equal concentrations: pan-caspase inhibitor (Z-VAD-FMK), caspase-3 inhibitor (ZDEVD-FMK), and caspase-9 inhibitor (Z-LEHD-FMK). Four birds received osmotic pumps filled with Z-FA-FMK, a negative control for caspase inhibitors $(0.015 \mathrm{mg}$ in $100 \mu \mathrm{l}$ in $1 \%$ DMSO). We placed the osmotic pumps into microcentrifuge tubes filled with saline, sealed the tubes with paraffin and quick-drying cement, and mounted the tubes into custom-made "backpacks." Eleven days after cannulae implantation, the pumps were swapped with fresh pumps filled with either caspase inhibitors or negative control, and the tubing was spliced with a stainless steel connector and cemented together with superglue.

To transition birds to nonbreeding conditions (SD photoperiod and withdrawal of circulating $\mathrm{T}$ ), we removed the subcutaneous $\mathrm{T}$-capsule $2 \mathrm{~d}$ after the cannula implantation. We shifted the birds overnight to $\mathrm{L} / \mathrm{D}=14 / 10$ on the same day as the T-withdrawal and shifted them overnight on the next day to SD. The intermediate photoperiod helped birds adjust to the reduction in available feeding time. We killed both groups of birds $20 \mathrm{~d}$ after photoshift and T-withdrawal.

Blood draw and hormone analysis. We took blood samples from the birds at various time points throughout the experiment to measure circulating $\mathrm{T}$ levels. We drew $250 \mu \mathrm{l}$ of blood from the alar vein in the wing into heparinized collection tubes. We immediately centrifuged the tubes to separate the plasma, which was stored at $-20^{\circ} \mathrm{C}$ until assay. We measured plasma $\mathrm{T}$ concentration using the Coat-A-Count Total Testosterone radioimmunoassay kit (Diagnostic Products). The minimum detectable plasma T concentration was $0.20 \mathrm{ng} / \mathrm{ml}$. Samples with undetectable levels of $\mathrm{T}$ were treated as having concentrations at this detection limit for statistical analysis. The intraassay and interassay coefficients of variation were $\sim 5$ and $12 \%$, respectively.

Kill, perfusion, tissue processing. We deeply anesthetized the birds with isoflurane inhalation and perfused them through the heart with heparinized saline followed by $4 \%$ phosphate-buffered paraformaldehyde (PFA). We postfixed the brains in $4 \%$ PFA for $24 \mathrm{~h}$, embedded the brains in gelatin, and postfixed the gelatin-embedded brains in a $20 \%$ sucroseneutral buffered formalin solution for $48 \mathrm{~h}$. We sectioned the brains in the coronal plane at $40 \mu \mathrm{m}$ on a freezing microtome, mounted every third section, and stained with thionin. We kept alternate sections for immunohistochemistry (see below).

Measurement of nuclei volume. Using an overhead projector, we traced the borders in both hemispheres throughout the full rostrocaudal extent of HVC. We scanned these tracings into a microcomputer and measured the surface area of each cross section using a customized module of NIH ImageJ (W. Rasband, National Institutes of Health, Bethesda, MD). We determined the volumes of nuclei by summing the estimated volume between sections calculated with the formula for the volume of a cone frustum (Tramontin et al., 1998). We excluded one bird infused with caspase inhibitors because of poor histology.

Measurement of neuronal attributes in HVC. We sampled neuron size by measuring the cross-sectional area of the soma in every Nissl-stained section throughout the rostral-caudal axis of HVC. We used a random, systematic sampling protocol that has been previously validated (Tramontin et al., 1998) and described in detail (Thompson and Brenowitz, 2008). Measurement of HVC neuron number in Nissl-stained sections and in sections stained for the neuron-specific marker protein $\mathrm{Hu}$ from the same brains in the closely related song sparrow yielded estimates that did not differ statistically (Tramontin and Brenowitz, 1999). We measured the area of the soma and density of at least $150 \mathrm{HVC}$ neurons per hemisphere of each bird in fields chosen randomly by computer in each section. We estimated neuron number by multiplying neuron density by total nucleus volume. Volume measurements and neuronal morphometry were made blind to whether birds were infused with caspase inhibitors or negative control.

$\mathrm{BrdU}$ and Hu immunohistochemistry. To determine the level of neuronal recruitment into HVC, we performed double-label immunohistochemistry for BrdU and $\mathrm{Hu}$. We placed sections in buffered glycerine and stored them in a $-80^{\circ} \mathrm{C}$ freezer until needed for immunohistochemistry. We rinsed floating sections containing contralateral and ipsilateral HVC in eight washes of $0.1 \mathrm{M} \mathrm{PBS}, \mathrm{pH}=7.4$. We transferred the sections to a $2 \mathrm{~N}$ hydrogen chloride bath held at $37^{\circ} \mathrm{C}$ for $30 \mathrm{~min}$ followed by a $10 \mathrm{~min}$ 
wash in $0.1 \mathrm{~m}$ Boric acid, $\mathrm{pH}=8.5$, at room temperature. We rinsed the sections in three washes of PBS, preblocked the tissue in $10 \%$ NGS for $1 \mathrm{~h}$, and incubated the tissue with primary antibodies against BrdU (PharMingen; 1:200) and $\mathrm{Hu}$ (Invitrogen; 1:500) diluted into in $10 \% \mathrm{NGS}$ overnight at $4^{\circ} \mathrm{C}$. We rinsed the sections in three washes of PBS and incubated them for $2 \mathrm{~h}$ in $10 \%$ NGS containing the secondary antibodies (Invitrogen; Alexa Fluor 488 $\alpha$-mouse, Alexa Fluor $594 \alpha$-rat; 1:200). Negative control tissue was prepared by omitting either the primary or the secondary antibody, which showed no positive staining. We mounted the sections onto slides, air dried them overnight, rehydrated them with $\mathrm{dH}_{2} \mathrm{O}$, and coverslipped them with Antifade mountant (Promega).

To quantify neuronal recruitment into HVC, we scanned the entire cross-sectional area of HVC and counted all cells within the border of HVC that showed positive nuclear labeling for $\mathrm{BrdU}$ and positive cytoplasmic labeling for $\mathrm{Hu}$. We confirmed that our counting protocol correctly identified cells that were double labeled using a Marianas imaging system (Intelligent Imaging Innovations) consisting of a CoolSnap HQ camera (Photometrics) and a Zeiss Axiovert 200M inverted microscope (Zeiss). The observer was blind to whether birds were infused with caspase inhibitors or negative control.

Statistics. We used a paired $t$ test $(\alpha<0.05)$ to compare ipsilateral to contralateral attributes.

\section{Results}

We compared measurements of HVC volume ipsilateral and contralateral to the infused hemisphere with paired $t$ tests ( $\alpha=$ 0.05 , two-tailed). Caspase inhibitor infusion protected ipsilateral HVC volume from regression $20 \mathrm{~d}$ after the transition to nonbreeding conditions ( $p=0.00613$ ); the mean ipsilateral HVC volume was $31.4 \%$ larger, on average, than the contralateral HVC volume (Fig. 2A). Infusion of a negative control had no effect on ipsilateral HVC volume ( $p=0.561)$, which was $2.5 \%$ larger than the contralateral HVC (Fig. $2 \mathrm{~A}$ ). Contralateral HVC volume in both treatment groups regressed as expected based on previous results (Thompson et al., 2007).

We measured HVC neuronal attributes from Nissl-stained sections using a random, systematic counting scheme (Tramontin et al., 1998). Caspase inhibitor infusion protected the ipsilateral HVC from neuron loss; mean ipsilateral neuron number was $29.7 \%$ greater than contralateral ( $p=0.00471$ ) (Fig. $2 B$ ). There was no difference in HVC neuron number ipsilateral and contralateral to infusion of a negative control $(p=0.844)$ (Fig. $2 B)$. Caspase inhibitor infusion had no effect on ipsilateral HVC soma area and neuron density ( $p=0.466$ and 0.513 , respectively) (Fig. $2 C, D)$, nor did the negative control infusion $(p=0.686$ and 0.155 , respectively) (Fig. 2C,D). HVC neuron density sharply increases hours after a transition to nonbreeding conditions, but then days to weeks later decreases to levels similar to those observed under breeding conditions (Thompson et al., 2007). Thus, the fact that caspase inhibitor infusion fails to have an effect on HVC neuron density when measured $20 \mathrm{~d}$ after transition to nonbreeding conditions is not unexpected.

We performed immunohistochemistry for BrdU and $\mathrm{Hu}$ to
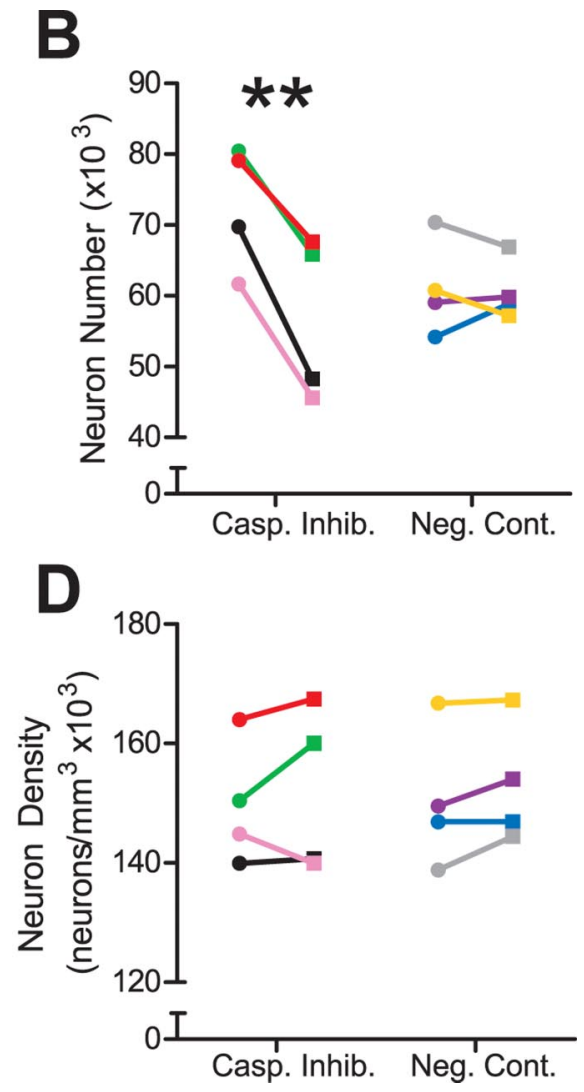

Figure 2. In vivo infusion of caspase inhibitors preserved ipsilateral HVC $20 \mathrm{~d}$ after the transition from breeding to nonbreeding

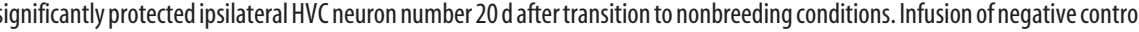
had no effect. $\boldsymbol{C}, \boldsymbol{D}$, Infusion of neither caspase inhibitors nor negative control significantly preserved ipsilateral HVC soma area and neuron density $20 \mathrm{~d}$ after the transition to nonbreeding conditions.

identify new neurons in sections containing ipsilateral and contralateral HVC (Fig. 3A-H). We injected birds with BrdU 22-27 $\mathrm{d}$ before kill, which is sufficient time for proliferation, migration, and recruitment of new neurons into HVC and for them to send projections to the robust nucleus of the arcopallium (RA) (Kirn et al., 1999; Scotto-Lomassese et al., 2007). Caspase inhibitor infusion significantly reduced the mean density and number of $\mathrm{BrdU} / \mathrm{Hu}$ positive neurons in the ipsilateral $\mathrm{HVC}$ by 47 and 29\%, respectively ( $p=0.00857$ and 0.0168 , respectively) (Fig. $3 I, J$ ); infusion of a negative control had no effect ( $p=0.913$ and 0.809 , respectively) (Fig. $3 I, J$ ). Given that unilateral syringeal nerve cut enhances neuronal recruitment into the contralateral HVC (Wilbrecht et al., 2002), it is possible that the differences we report here could be a result enhanced neuronal recruitment into the contralateral HVC. Although the results from one animal infused with caspase inhibitors (Fig. $3 \mathrm{I}$, J, red symbols) seem to indicate that this may be the case, new neuron density and number on average are 30.3 and $13.3 \%$ lower, respectively, in the ipsilateral HVCs of birds infused with caspase inhibitors versus negative control, which indicates that caspase inhibitor infusion reduces recruitment to the ipsilateral HVC instead of enhancing recruitment to the contralateral HVC. It should be noted that these differences were not statistically different in an ANOVA, however. We believe that this lack of significance is caused by two factors: (1) the test power is too low for this analysis (both results have a power of 0.050 , well below the threshold value of 0.80 at 

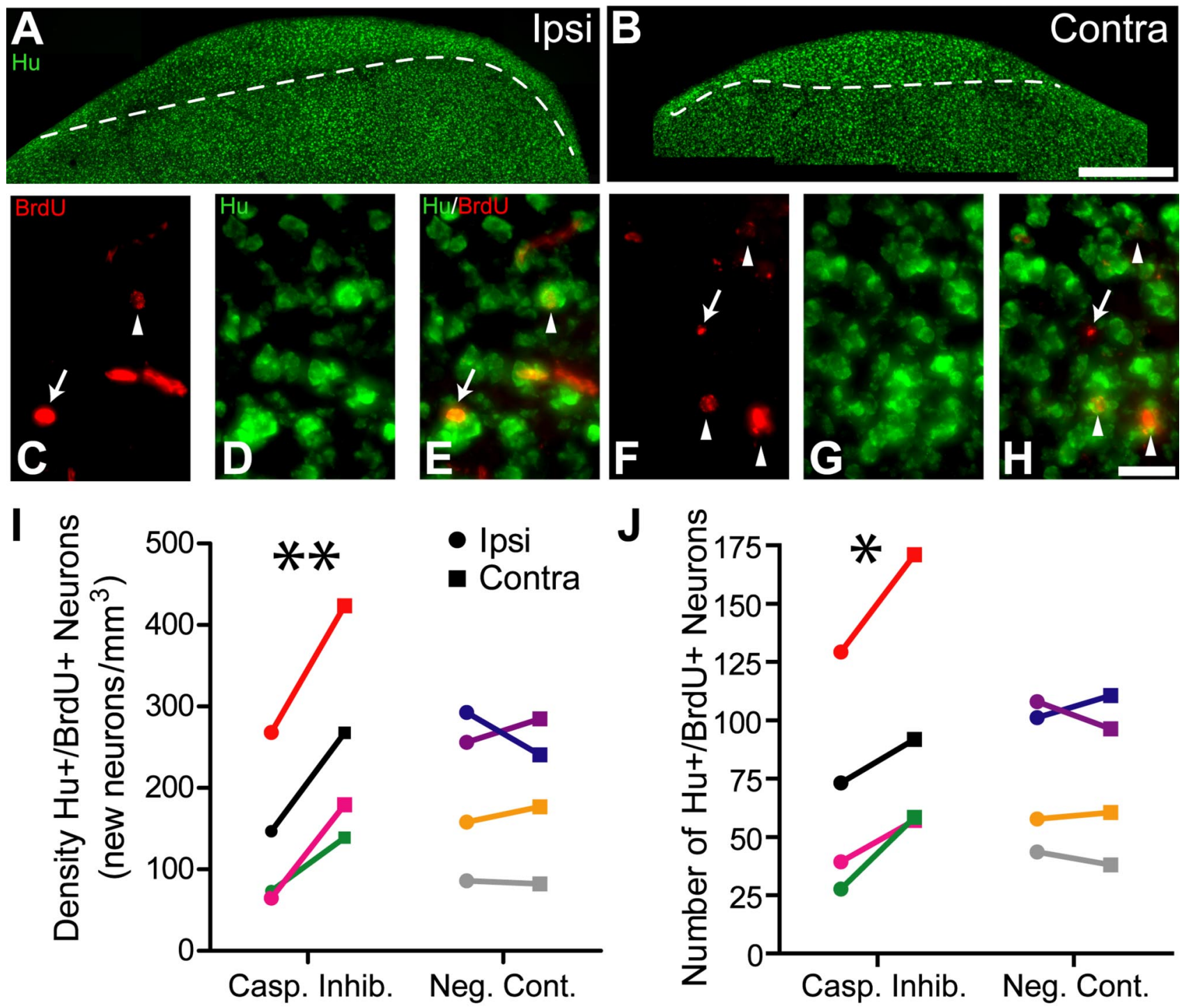

Figure 3. In vivo infusion of caspase inhibitors near HVC significantly reduced the rate of incorporation of new neurons into the ipsilateral HVC $20 \mathrm{~d}$ after the transition from breeding to nonbreeding conditions. $\boldsymbol{A}, \boldsymbol{B}$, Micrographs illustrating immunohistochemistry for $\mathrm{Hu}$, a neuron-specific marker in a section containing $\mathrm{HV} \boldsymbol{C}$ on the ipsilateral $(\boldsymbol{A})$ and contralateral $(\boldsymbol{B})$ hemisphere, show that caspase inhibitor infusion prevented the regression of the ipsilateral HVC, while the contralateral HVC regressed normally. Dashed lines indicate the ventral border of HVC. Scale bar, 200 $\mu \mathrm{m} . \boldsymbol{C}-\boldsymbol{H}$, High-magnification micrographs of cells positive for $\operatorname{BrdU}(\boldsymbol{C}, \boldsymbol{F})$ and $\mathrm{Hu}(\boldsymbol{D}, \boldsymbol{G})$, and merge for both labels $(\boldsymbol{E}, \boldsymbol{H})$ depict fewer BrdU $+/$ Hu + neurons (arrowheads) in the ipsilateral $\mathrm{HVC}$ $(\boldsymbol{E})$ than in the contralateral HVC $(\boldsymbol{H})$. Brd $+/ \mathrm{Hu}$ - cells are indicated with an arrow. Scale bar, $20 \mu \mathrm{m}$. $\boldsymbol{I}, \boldsymbol{J}$, Caspase inhibitor infusion significantly reduced the density $(\boldsymbol{I})$ and number $(\boldsymbol{J})$ of new neurons incorporated into the ipsilateral HVC. Infusion of a negative control had no effect. Asterisk indicates significant differences across hemispheres (pairwise $t$ test; ${ }^{*} p<0.05$, ${ }^{* *} p<0.01$ ).

which one can be confident in interpreting negative results such as these), and (2) as noted above, the results from the caspase inhibitor infused bird illustrated in red had particularly high values for new neuron density and number, which increased the variability substantially. Nevertheless, our study was designed to emphasize paired comparisons between the ipsilateral and contralateral sides of HVC within individual birds, not between birds from different treatment groups.

We used radioimmunoassay to measure circulating levels of T in blood samples taken while birds were under breeding and nonbreeding conditions. When birds were under breeding conditions, T levels were $11.06 \pm 0.99 \mathrm{ng} / \mathrm{ml}(\mathrm{X} \pm \mathrm{SEM}, n=9)$. We also collected plasma at kill, $20 \mathrm{~d}$ after castration $(0.73 \pm 0.21$ $\mathrm{ng} / \mathrm{ml}, n=9$. The measured level of circulating $\mathrm{T}$ from one bird under breeding conditions was excluded from analysis because of insufficient volume of plasma collected. The measured level of circulating $\mathrm{T}$ from one bird in the negative control group under nonbreeding conditions was also excluded from hormone analysis because it was substantially higher than expected for castrated birds on SD. The total volume of plasma collected at kill for this bird was less than optimal, which may have contributed to the anomalous value. Statistical analysis indicates that this datum is an outlier (i.e., > mean $+2 \mathrm{SD}$ ). Given that this bird had neither testes nor a subcutaneous T-pellet at kill, and neuronal attributes measured for this bird were typical of other birds killed under nonbreeding conditions, the measurements of neuronal attributes from this bird were included in the results. Omitting the hormone data from this bird did not change the outcome of statistical analysis, which showed that levels of circulating $\mathrm{T}$ were significantly reduced ( $p<0.001, n=8$, paired $t$ test).

\section{Discussion}

Our study has two major results. (1) Infusion of caspase inhibitors is sufficient to protect neurons in a brain area that would 
otherwise die at least $20 \mathrm{~d}$ after onset of neurodegeneration. (2) Blockade of neuron loss reduces the rate of neuronal recruitment into a brain area that undergoes neuron turnover. Together, these results show that neuron death is necessary for subsequent neuron recruitment.

Our results directly demonstrate that neuron death is necessary for neuronal recruitment, which is consistent with results from other studies. In adult male canaries seasonal increases in the rate of neuronal recruitment into HVC is preceded by increases in cell death 2 months prior (Kirn et al., 1994). In adult male song sparrows, neuronal recruitment into HVC is highest in the fall, when HVC is fully regressed (Tramontin and Brenowitz, 1999). Although these results are largely correlational, the sufficiency of neuron death to induce increased neuronal recruitment has been tested in other studies. In adult male zebra finch HVC (Scharff et al., 2000) and adult female mouse cortex (Magavi et al., 2000; Chen et al., 2004), chromophore-targeted photolytic neuronal degeneration increases the rate of neuronal recruitment into existing brain circuits. Ischemia induces neurogenesis in the CA1 region of the hippocampus (Nakatomi et al., 2002) and in the striatum (Arvidsson et al., 2002). Infusion of a caspase inhibitor into the dentate gyrus (DG) of adult mice reduces the rate of DG cell death and reduces the rate of recruitment of new cells into the DG (Dupret et al., 2007). At least some of these new cells were likely to be neurons, although Dupret et al. (2007) did not use a neuron-specific label to conclusively demonstrate that they were newly recruited neurons.

The fact that caspase inhibitor infusion reduced the rate of neuronal recruitment is particularly striking given that one might expect that caspase inhibitors would increase the survival of new neurons in HVC, many of which normally die within the first $30 \mathrm{~d}$ after migrating to HVC (Kirn et al., 1999). If that were the case, then we would expect to see more new neurons in the ipsilateral HVC compared with the contralateral HVC. Instead, caspase inhibitor infusion led to fewer new neurons in the ipsilateral HVC over the $20 \mathrm{~d}$ time period. Caspase inhibitor infusion prevented the loss of HVC neurons that would normally die after the transition to nonbreeding conditions, which would have resulted in fewer "vacancies" for new neurons in the ipsilateral HVC. A vacancy may represent newly available synaptic space and/or increased uptake/release of trophic factor that may result from neuron death.

We showed that in vivo infusion of caspase inhibitors for longer than 1 week can protect neurons that would otherwise die. This observation is especially noteworthy given that there is some evidence that cells that would normally die via activation of caspases may resort to caspase-independent cell death pathways if caspase activity is persistently inhibited (Furre et al., 2006). Caspase inhibitors did not protect HVC soma area from regression in the current study, although the results from two animals seem to indicate some protection of ipsilateral HVC soma area (Fig. 2C). This result is surprising given that in a previous study caspase inhibitors reduced the regression of HVC soma area at $7 \mathrm{~d}$ after a transition to nonbreeding conditions (Thompson and Brenowitz, 2008). This may be because of several factors, including the fact that HVC soma area varies only minimally across seasons, particularly in comparison to the changes observed in RA soma area. Also, the effectiveness of caspase inhibitors in preventing cleavage of cytoskeletal structural elements required for an increase in soma area may have decreased toward the end of their infusion. Nevertheless, our data indicate that the mixture of caspase inhibitors used in this study continues to effectively protect HVC volume and neuron number for at least $20 \mathrm{~d}$ in vivo after the transition to nonbreeding conditions.

Several mechanisms may mediate the relationship between apoptosis and neurogenesis. In mammals, neuron death increases expression of various trophic factors, including vascular epithelial growth factor (VEGF) and brain derived neurotrophic factor (BDNF), in brain circuits undergoing neurodegeneration (Hayashi et al., 1997; Wang et al., 1998). Trophic factors can increase the rate of neuronal proliferation and differentiation, enhance survival during migration and recruitment, and increase axonal and dendritic arborization and synapse formation, which facilitate incorporation (Ming and Song, 2005). Locally enhanced trophic factor availability may allow for increased rates of neuronal incorporation to fill synaptic vacancies created by the death of mature neurons. In adult female canaries, in vivo infusion of BDNF into HVC increases the rate of neurogenesis, and blockade of BDNF prevents the $\mathrm{T}$-induced increase in neurogenesis (Rasika et al., 1999). In addition, in vivo inhibition of a tyrosine kinase associated with a receptor for VEGF significantly reduces the rate of neurogenesis (Louissaint et al., 2002). If similar mechanisms mediate neurogenesis after the transition from breeding to nonbreeding conditions, then we would predict that (1) the loss of HVC neurons in adult male white-crowned sparrows may lead to transient increased expression of trophic factors such as BDNF and VEGF, and (2) caspase inhibitor infusion may block an increase in the expression of trophic factors by preventing neuron loss.

Adult neurogenesis is a multistep process comprised of proliferation, differentiation, migration, and incorporation of new neurons into existing brain circuits. The prevention of neuron death via caspase inhibitor infusion may influence the rate of any or all of these steps. Knocking out Bax, a pro-apoptotic factor critical for programmed cell death, in adult mice reduces neuronal turnover in the olfactory bulb, which ultimately leads to severely disrupted migration of neuronal precursors in the rostral migratory stream (Kim et al., 2007). BrdU+ neurons in our study were $4-8$-d-old at the onset of nonbreeding conditions and were most likely still in the process of migrating from the ventricular zone into HVC at the time of transition (Kirn et al., 1999). If caspase inhibitor-mediated protection of HVC neurons from programmed cell death reduces the rate of migration of new neurons into HVC, then newborn neurons might accumulate in the ventricular zone or migrate to other parts of the nidopallium. Last, although our results suggest that caspase inhibitor infusion must have some impact on neuronal migration, we cannot rule out effects on rates of neuronal proliferation and differentiation as well.

We showed that caspase inhibitor infusion in vivo protects HVC from neurodegeneration for as long as $20 \mathrm{~d}$ after the transition to nonbreeding conditions. The reduced rate of neuron death in HVC decreased available vacancies for new neurons to be incorporated into HVC. Our results show that an increased rate of neuron death is necessary for a seasonally increased rate of neuronal incorporation in adult brain circuits that undergo constant neuronal turnover.

\section{References}

Altman J, Das GD (1965) Autoradiographic and histological evidence of postnatal hippocampal neurogenesis in rats. J Comp Neurol 124:319-335.

Arvidsson A, Collin T, Kirik D, Kokaia Z, Lindvall O (2002) Neuronal replacement from endogenous precursors in the adult brain after stroke. Nat Med 8:963-970.

Brenowitz EA, Nalls B, Wingfield JC, Kroodsma DE (1991) Seasonal 
changes in avian song nuclei without seasonal changes in song repertoire. J Neurosci 11:1367-1374.

Chen J, Magavi SS, Macklis JD (2004) Neurogenesis of corticospinal motor neurons extending spinal projections in adult mice. Proc Natl Acad Sci U S A 101:16357-16362.

Dupret D, Fabre A, Döbrössy MD, Panatier A, Rodríguez JJ, Lamarque S, Lemaire V, Oliet SH, Piazza PV, Abrous DN (2007) Spatial learning depends on both the addition and removal of new hippocampal neurons. PLoS Biol 5:e214.

Furre IE, Møller MT, Shahzidi S, Nesland JM, Peng Q (2006) Involvement of both caspase-dependent and -independent pathways in apoptotic induction by hexaminolevulinate-mediated photodynamic therapy in human lymphoma cells. Apoptosis 11:2031-2042.

Goldman SA, Nottebohm F (1983) Neuronal production, migration, and differentiation in a vocal control nucleus of the adult female canary brain. Proc Natl Acad Sci U S A 80:2390-2394.

Hayashi T, Abe K, Suzuki H, Itoyama Y (1997) Rapid induction of vascular endothelial growth factor gene expression after transient middle cerebral artery occlusion in rats. Stroke 28:2039-2044.

Kim WR, Kim Y, Eun B, Park OH, Kim H, Kim K, Park CH, Vinsant S, Oppenheim RW, Sun W (2007) Impaired migration in the rostral migratory stream but spared olfactory function after the elimination of programmed cell death in bax knock-out mice. J Neurosci 27:14392-14403.

Kirn J, O’Loughlin B, Kasparian S, Nottebohm F (1994) Cell death and neuronal recruitment in the high vocal center of adult male canaries are temporally related to changes in song. Proc Natl Acad Sci U S A 91:7844-7848.

Kirn JR, Fishman Y, Sasportas K, Alvarez-Buylla A, Nottebohm F (1999) Fate of new neurons in adult canary high vocal center during the first 30 days after their formation. J Comp Neurol 411:487-494.

Louissaint A Jr, Rao S, Leventhal C, Goldman SA (2002) Coordinated interaction of neurogenesis and angiogenesis in the adult songbird brain. Neuron 34:945-960.

Magavi SS, Leavitt BR, Macklis JD (2000) Induction of neurogenesis in the neocortex of adult mice 405:951-955.

Ming GL, Song H (2005) Adult neurogenesis in the mammalian central nervous system. Annu Rev Neurosci 28:223-250.

Nakatomi H, Kuriu T, Okabe S, Yamamoto S, Hatano O, Kawahara N, Tamura A, Kirino T, Nakafuku M (2002) Regeneration of hippocampal pyramidal neurons after ischemic brain injury by recruitment of endogenous neural progenitors. Cell 110:429-441.

Paton JA, Nottebohm FN (1984) Neurons generated in the adult brain are recruited into functional circuits. Science 225:1046-1048.

Rasika S, Alvarez-Buylla A, Nottebohm F (1999) BDNF mediates the effects of testosterone on the survival of new neurons in an adult brain. Neuron 22:53-62.

Scharff C, Kirn JR, Grossman M, Macklis JD, Nottebohm F (2000) Targeted neuronal death affects neuronal replacement and vocal behavior in adult songbirds. Neuron 25:481-492.

Scotto-Lomassese S, Rochefort C, Nshdejan A, Scharff C (2007) HVC interneurons are not renewed in adult male zebra finches. Eur J Neurosci 25:1663-1668.

Smith GT, Brenowitz EA, Wingfield JC, Baptista LF (1995) Seasonal changes in song nuclei and song behavior in Gambel's white-crowned sparrows. J Neurobiol 28:114-125.

Thompson CK, Brenowitz EA (2008) Caspase inhibitor infusion protects an avian song control circuit from seasonal-like neurodegeneration. J Neurosci 28:7130-7136.

Thompson CK, Bentley GE, Brenowitz EA (2007) Rapid seasonal-like regression of the adult avian song control system. Proc Natl Acad Sci U S A 104:15520-15525.

Tramontin AD, Brenowitz EA (1999) A field study of seasonal neuronal incorporation into the song control system of a songbird that lacks adult song learning. J Neurobiol 40:316-326.

Tramontin AD, Smith GT, Breuner CW, Brenowitz EA (1998) Seasonal plasticity and sexual dimorphism in the avian song control system: stereological measurement of neuron density and number. J Comp Neurol 396:186-192.

Tramontin AD, Hartman VN, Brenowitz EA (2000) Breeding conditions induce rapid and sequential growth in adult avian song control circuits: a model of seasonal plasticity in the brain. J Neurosci 20:854-861.

Wang Y, Sheen VL, Macklis JD (1998) Cortical interneurons upregulate neurotrophins in vivo in response to targeted apoptotic degeneration of neighboring pyramidal neurons. Exp Neurol 154:389-402.

Wilbrecht L, Petersen T, Nottebohm F (2002) Bilateral LMAN lesions cancel differences in HVC neuronal recruitment induced by unilateral syringeal denervation. Lateral magnocellular nucleus of the anterior neostriatum. J Comp Physiol A Neuroethol Sens Neural Behav Physiol 188:909915. 